\title{
APPLICATION OF SELECTIVE LEACHING IN FABRICATION OF THIN FILM YBCO DEVICES.
}

\author{
P.M. James, J.M. O'Callaghan and A.B. Ellis. \\ University of Wisconsin - Madison ${ }^{1}$.
}

\begin{abstract}
Ethylenediamine solutions have been shown to turn bulk YBCO into insulating materials. In this work, the effect of these solutions on thin YBCO films is studied. In both unpatterned and patterned films, a smooth decrease in critical currents, a transition to normal state and a subsequent gradual increase in resistance is observed as a function of exposure time to the solution. These characteristics might make this process desirable for weak link and on-film resistor fabrication.
\end{abstract}

\section{INTRODUCTION}

Some thin film device fabrication processes require controlled weakening of the superconductor $[1,2]$. Turning superconductor into resistive material is also of use in thin film circuit fabrication when on-film resistors or RF matched loads are required. In this work we assess the usefulness of ethylenediamine solutions to achieve both effects.

These solutions have already been shown to preferentially leach the copper from bulk YBCO samples, leaving behind materials known to be insulating [3].

When YBCO films are exposed to such solutions, leaching creates a layer whose copper concentration has been reduced from its original value. Auger profiles have shown that the relative concentration of copper in a partially leached film increases gradually from a minimum value at the surface to a constant, stoichiometric value at some depth within the film. This depth is dependent on the exposure of the film to the ethylenediamine solution. The combined effect of material modification and reduction of the thickness of the superconducting layer results in a reduction of the critical current and an increase in resistance once the sample is no longer superconductive.

A potential advantage of this process with respect to the conventional method of weak link fabrication (etching film away in the weak link regions $[1,4,5])$ is enhanced controllability, since as unlike for etching, diffusion tends to slow down the leaching process as deeper film layers are chemically modified.

${ }^{1}$ P.M. James and A.B. Ellis are with the department of Chemistry 1101 University Av. Madison WI. 53706

J.M. O'Callaghan is with the department of Electrical and Computer Engineering, 1415 Johnson Dr. Madison WI 53706.

This work is supported by NSF throught a Materials Research Group Grant and by the Spanish Ministry of Education and Science.

Manuscript received August 24, 1992

\section{EXPERIMENTAL}

\section{A. Materials}

Films from several sources were used in this work and wide variations in reactivity were observed from film to film. Most of the films were prepared by Bruce Davidson at the University of Wisconsin, Madison by laser ablation on $\mathrm{LaAlO}_{3}$ and $\mathrm{MgO}$ substrates. These films produced the most repeatable results.

Olin Hunt HR-100 negative PR and Hoechst Celanese $A Z 1350 J$ positive $P R$ were used for patterning and later processing. The leaching solution was prepared from ethylenediamine (Aldrich $99 \%$ ethylenediamine -- used as shipped) and triply distilled water as a $2.0 \mathrm{M}$ solution and used at room temperature. Contacts were made by sputter depositing silver, which was later annealed in oxygen (1 atm) at $375^{\circ} \mathrm{C}$ for $60 \mathrm{~min}$. Epo-Tek $\mathrm{H} 31$ silver epoxy, cured for 4 hr at $100^{\circ} \mathrm{C}$, was used to bond 36 gauge copper wires to the pads.

\section{B. Procedures}

Mechanical tests: Thickness versus leach experiments where performed on several patterned films using profilometry. Measurement error was highest in films with $\mathrm{LaAlO}_{3}$ substrates due to the terraces formed by twinning. No appreciable change in film thickness was seen in any of the films measured, as shown in Fig. 1. This was also confirmed by SEM images.

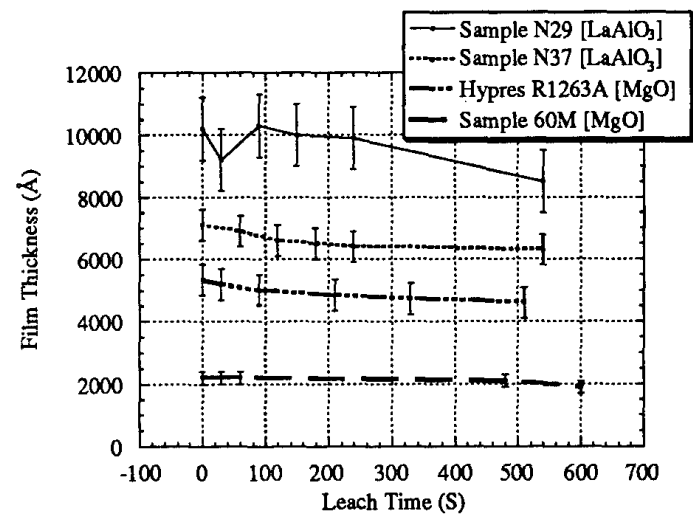

Fig. 1 Thickness vs. exposure to $2.0 \mathrm{M}$ ethylenediamine solution for several different YBCO films, as measured by profilometry. 
Electrical tests in unpatterned films: The critical current and critical temperature were measured in several unpatterned films using a 4 point probe setup. Figures 2 and 3 show a typical set of such results. Smooth transitions from metallic to insulating behavior were observed above the critical temperature in the transition diagrams as the leaching time was increased, and no significant changes in critical temperature were found as long as the sample remained superconductive (a resistivity drop was still detected when samples were not fully superconducting). Below $T_{C}$, the critical currents were also gradually depressed as the films were exposed to the leaching solutions. Eventually, the films became normal conductors. Once this happened, their resistivity could be gradually increased by continuing the exposure of the films to the leachant. Such increases could be carried out over many orders of magnitude.

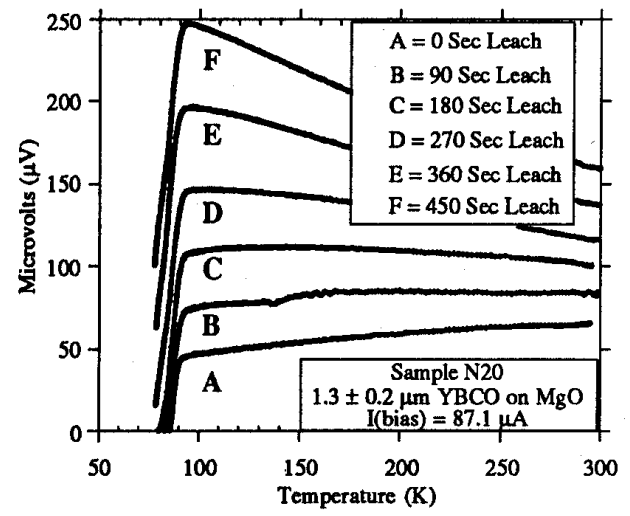

Fig. 2 Transition diagram of an unpatterned film with leaching time as a parameter.

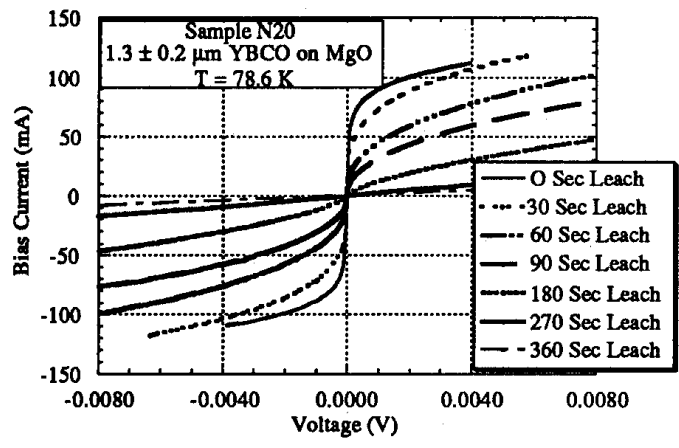

Fig. 3 I-V Curves of an unpatterned with leaching time as a parameter.

Electrical tests in patterned films: Films were patterned using AZ1350J photoresist and a $\mathrm{HNO}_{3}$ solution [1]. Six links of varying sizes were patterned in each film, and the films were covered with HR-100 resist with openings on the links and contact areas. This resist withstands thermal cycling, which allows leaching and testing of the samples in cyclic sequence.

Preliminary results show transition diagrams and I-V curves qualitatively comparable to Figs. 2 and 3 . Comparison among links in the same film is indicated in Fig. 4.

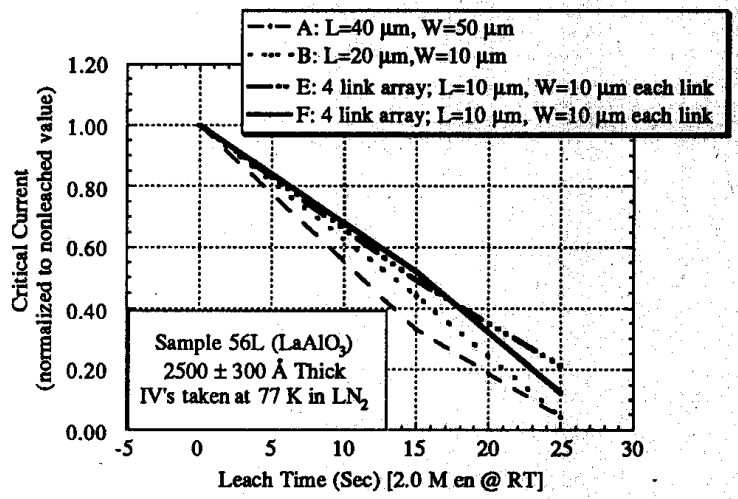

Fig. 4 Relative reduction in critical current of four links of varying lengths $(\mathrm{L})$ and widths $(W)$ pattemed in the same film.

At this point it is suspected that residues from silver paint solvents are deposited in the links after each leaching stage, and they interfere with the leaching process. Such residues could come from the silver paint (used to attach the film substrate to a copper block) dissolving when the sample is rinsed with methanol after leaching. It is hoped that greater uniformity throughout the film will be obtained once this is solved.

\section{CONCLUSION}

Ethylenediamine solutions are being tested in thin film device applications. Results on unpatterned films indicate adequate behavior of these solutions for weak link and onfilm resistor fabrication. This has been qualitatively reproduced in patterned films. Achieving uniformity in the characteristics of several links within a film still presents practical problems which are currently being addressed.

\section{ACKNOWLEDGMENT}

The authors are thankful to Bruce Davidson for preparing most of the YBCO films used in this work. Gert Hohenwarter also provided films produced by Hypres Inc. Films made by Bruce Lairson at Stanford University were also used.

\section{REFERENCES}

[1] J.S. Martens, D.S. Ginley, J.B. Beyer, J.E. Nordman, G.K.G. Hohenwarter. "A Model and Equivalent Circuit 
for a Superconducting Flux Flow Transistor". IEEE Trans. on Applied Superconductivity. Vol. 1, No. 2 June 1991, pp 95-101.

[2] J.S. Martens , V.M. Hietala, T.E. Zipperian, D.S. Ginley, C.P. Tigges, J. M. Phillips. "S Parameter Measurements and Microwave Applications of Superconducting Flux Flow Transistors". IEEE Trans. on Microwave Theory and Techniques. Vol. 39, No. 12, December 1991, pp 1231-1234.

[3] P.M. James, E.J. Thompson, A.B. Ellis. "Selective Leaching of Copper and Barium from the YBCO Superconductor by Chelating Diamines". Chemistry of Materials, Vol. 3, No. 6, 1991, pp. 1087-1092.
[4] F.K. Shokoohi, L.M. Schiavone, C.T. Rodgers, A. Inam X.D. Wu, L Nazar and T. Venkatesan. "Wet Chemical Etching of High Temperature Superconducting YBCO Films in Ethylenediaminetetraacetic Acid". Applied Physics Letters, Vol. 55, No. 25 , Dec. 1989, pp. 2661 2663.

[5] C.I.H. Ashby, J. Martens, T.A. Plut and D.S. Ginley."Improved Aqueous Etchant for High Tc Superconductor Materials". Applied Physics Letters, Vol. 60, No. 17, April 1992, pp. 2147-2149. 